\title{
A retrospective observational study of benign anthracotic lymphadenitis and its association with PET avid lymph nodes in patients undergoing cancer evaluation
}

\author{
Nathaniel M. Ivanick ${ }^{1,2}$, Pavan Shrestha ${ }^{1}$, Michael J. Podolsky ${ }^{1}$, Vighnesh Walavalkar ${ }^{3}$, \\ Calixto-Hope Lucas ${ }^{3}$, Yaron B. Gesthalter ${ }^{1}$, Eric J. Seeley ${ }^{1}$ \\ ${ }^{1}$ Department of Medicine, Division of Pulmonary and Critical Care Medicine, University of California, San Francisco, CA, USA; ${ }^{2}$ Department \\ of Thoracic Surgery, Roswell Park Comprehensive Cancer Center, Buffalo, NY, USA; ${ }^{3}$ Department of Pathology, University of California, San \\ Francisco, CA, USA \\ Contributions: (I) Conception and design: EJ Seeley, MJ Podolsky; (II) Administrative support: EJ Seeley, NM Ivanick, P Shrestha; (III) Provision of \\ study materials or patients: EJ Seeley, YB Gesthalter; (IV) Collection and assembly of data: EJ Seeley, NM Ivanick, P Shrestha, V Walavalkar, CH \\ Lucas; (V) Data analysis and interpretation: EJ Seeley, NM Ivanick, P Shrestha, V Walavalkar, CH Lucas, YB Gesthalter; (VI) Manuscript writing: \\ All authors; (VII) Final approval of manuscript: All authors. \\ Correspondence to: Eric J. Seeley, MD. Department of Medicine, University of California, San Francisco, HSE 1355, Box 0111, 505 Parnassus Ave, San \\ Francisco, CA 94143, USA. Email: Eric.seeley@ucsf.edu.
}

Background: Accurate staging of newly diagnosed or recurrent malignancy is essential for effective treatment. An important first step in staging involves the use of PET/CT to identify areas of FDG avidity. PET/CT however has limitations, including false positive FDG uptake from benign causes. In this paper we characterize an uncommon yet clinically important cause of false positive PET/CTs, that of benign anthracotic lymphadenitis (BAL). We examine the clinical, radiographic and histologic characteristics of BAL in patients referred for endobronchial ultrasound (EBUS) guided biopsies and discuss its context in relation to existing literature.

Methods: We performed a retrospective observational case series of 20 patients who were referred for EBUS guided biopsies of PET positive mediastinal and hilar lymph nodes during the work-up or treatment of suspected malignancy.

Results: To be included, all patients received PET imaging as well as an EBUS guided biopsy of FDG avid lymph nodes which demonstrated anthracotic pigment as the only histologic abnormality. The key findings were that $90 \%$ of patients in this cohort were born outside of the US, $90 \%$ had bilateral FDG avid lymph nodes with an average standardized uptake value (SUV) of 7.9 2.2. Most patients, based on their history, had a likely exposure to biomass fuel or urban pollution.

Conclusions: BAL may be an underrecognized cause for PET positive lymph nodes in patients undergoing work-up for malignancy. These findings support the importance of sampling mediastinal and hilar lymph nodes even when SUVs are highly suggestive of malignancy.

Keywords: Anthracosis; endobronchial ultrasound (EBUS); mediastinal lymphadenopathy; non-small cell lung cancer (NSCLC); PET scan; PET/CT fusion imaging

Submitted Jan 23, 2021. Accepted for publication May 27, 2021.

doi: $10.21037 /$ jtd-21-142

View this article at: https://dx.doi.org/10.21037/jtd-21-142

(C) Journal of Thoracic Disease. All rights reserved. 


\section{Introduction}

PET scans and more recently combined PET/CTs have become an essential step in the staging of suspected or biopsy proven malignancies. PET imaging is now the guideline recommended initial imaging study to assess for metastatic disease in biopsy proven lung, esophageal and head and neck cancers (1-3). In addition, it is an essential tool for staging in lymphoma (4). In the evaluation of nodal metastases or distant metastatic disease in lung cancer, $\mathrm{PET} / \mathrm{CT}$ is more sensitive than CT alone and meta-analysis suggest a pooled sensitivity of $62 \%$, with a pooled specificity of $90 \%$ (1). The increasing use of PET/CT imaging has coincided with the development and widespread use of endobronchial ultrasound (EBUS) as a tool to histologically sample the hilar and mediastinal lymph nodes in order to invasively stage patients with newly diagnosed lung cancer and malignancies that metastasize to the thorax (5). This co-evolution has provided an ideal opportunity to characterize causes of "false positive" PET/CTs. "False positive" PET/CT's are noted in a number of metabolically active benign diseases that manifest increased FDG uptake. These alternative conditions include infections such as mycobacterial and fungal infections, as well as inflammatory conditions, such as sarcoidosis (6). A poorly characterized and less commonly appreciated cause of positive PET/CTs is benign anthracotic lymphadenitis (BAL) (7-9).

In this case series, we describe the demographic, imaging and histologic findings in a series of patients with BAL. As geographical borders in medical care dissolve, attention to causes false positive PET/CT imaging will need to be reevaluated in order to effectively prevent non-invasive over-staging of lung and other cancers.

We present the following article in accordance with the STROBE reporting checklist (available at https://dx.doi. org/10.21037/jtd-21-142).

\section{Methods}

\section{Patient selection}

All patients were referred to the interventional pulmonary clinic at the UCSF medical center for evaluation of PET avid mediastinal and/or hilar lymphadenopathy. In order to be included in this retrospective observational case series, all patients had a ${ }^{18} \mathrm{~F}$-FDG PET or ${ }^{18} \mathrm{~F}$-FDG PET/ $\mathrm{CT}$ prior to undergoing EBUS guided tissue sampling. All patients with a final pathological diagnosis of anthracotic pigment between December 15, 2016 and July 25, 2018 were included. All patients had a mediastinal or hilar lymph node with a PET standardized uptake value $(\mathrm{SUV})>2.5$. Demographic data, including age, gender, country of origin, past oncologic history and smoking history were recorded for each patient. Six patients had surgical resection of lymph nodes that were biopsied with EBUS as part of their surgical management for lung cancer.

\section{Imaging characteristics}

Data on ${ }^{18} \mathrm{~F}$-FDG PET/CT or ${ }^{18} \mathrm{~F}$-FDG PET were obtained from review of imaging and radiology reports. Lymph nodes stations were defined according to the International Association for the Study of Lung Cancer (IASLC) lymph node map (10). SUV of individual lymph nodes were obtained from the radiology report. The lymph node with the highest SUV was reported and only lymph nodes with SUV greater than 2.5 were considered positive (11).

Selection of lymph node for biopsy and number of lymph nodes biopsied was determined by review of the ${ }^{18} \mathrm{~F}-\mathrm{FDG}$ PET/CT or ${ }^{18} \mathrm{~F}$-FDG PET and the clinical context and was at the discretion of the attending pulmonologist. For staging procedures, the highest nodal station was biopsied first.

\section{Specimen collection}

All patients underwent an EBUS guided bronchoscopic biopsy (BF-UC190F, Olympus, Tokyo, Japan) under general anesthesia with either a laryngeal mask airway or endotracheal tube at the University of California, San Francisco, Medical Center. An Olympus EBUS bronchoscope (BF-UC190F, Olympus, Tokyo, Japan) with a 21 or $22 \mathrm{~g}$ needle was used for all biopsies (ViziShot EBUS TBNA Needle, Olympus, Tokyo, Japan). Onsite cytology was present for all biopsies to confirm adequacy of the specimens. Lymph node specimens were considered as positive for benign anthracotic inflammation if one or more lymph nodes in the mediastinum or hilum had an SUV $>2.5$ and the biopsy specimen showed anthracotic pigment (black pigment) inside histiocytes and macrophages, as interpreted by the attending pathologist (Figure 1).

\section{IRB approval}

The study was conducted in accordance with the Declaration of Helsinki (as revised in 2013). The study 

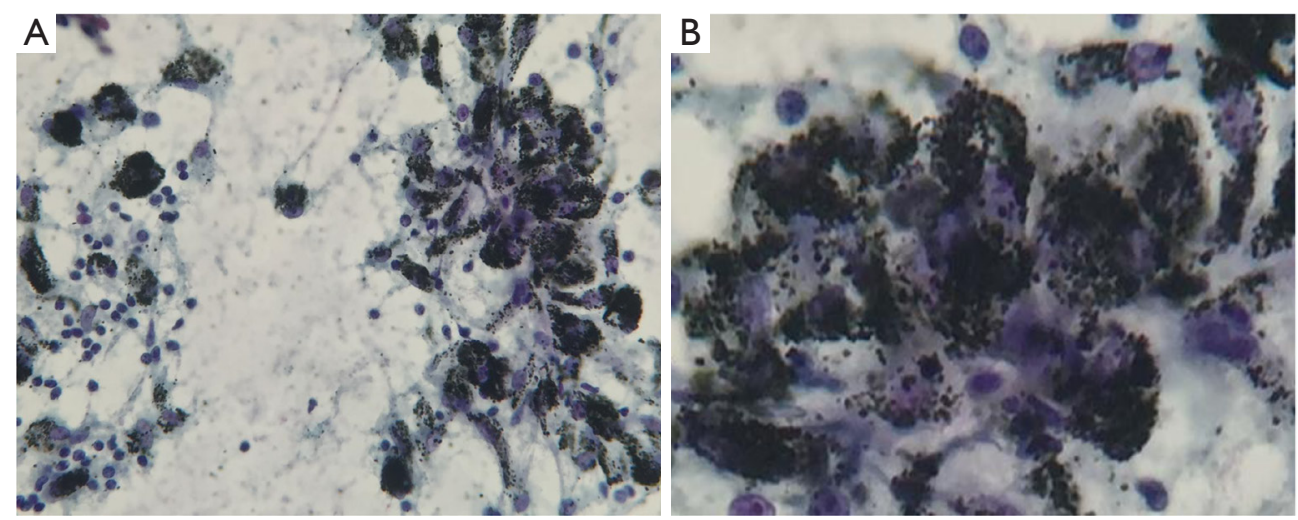

Figure 1 Papanicolaou stained smears of the station 11R lymph node fine needle aspiration showed numerous pigment filled macrophages in a background of mature lymphocytes, fibrosis and granulation tissue. There was no evidence of metastatic carcinoma or of lymphoma. (A,B) Cytology 20x magnification.

was approved by institutional ethics committee of University of California San Francisco Office of Ethics and Compliance (IRB \#17-23634) and individual consent for this retrospective study was waived.

\section{Statistical analysis}

Data was collected prospectively using REDCAP. All data analysis was performed using STATA. Lymph node size and SUV measurement were obtained from the index PET/CT scan. Lymph node size was measured by the authors in short axis diameter and SUV was taken from the radiologists quantitation. Data are displayed as mean \pm standard deviation. As this was a retrospective observational study, there was no pre-determined study size considered. No patients were lost to follow up.

\section{Results}

Demographic characteristics of enrolled subjects are summarized in Table 1 and are notable for advanced age ( $73 \pm 10.1$ years), male predominance $(70 \%)$, heavy smoking exposure $(50 \%)$ and birth outside of the United States (90\%). Patients came from countries on multiple continents, including Asia/Asia subcontinent, North and Central America and Middle Eastern/Near Eastern countries (Table 1). Exposure history was varied and included dust exposure from outdoor occupations in construction and landscaping. Other patients were exposed to indoor cooking using biomass fuels in poorly ventilated homes (12). Information on potential source of exposure was obtained from review of the medical record.

In patients with known malignancy, non-small cell lung cancer (NSCLC) was the most heavily represented lung cancer, followed by gastrointestinal, head and neck, breast and genitourinary cancers. Two patients had non-epithelial derived malignancies (one with MALT lymphoma and one with Myxoid Liposarcoma). Three patients did not have an underlying malignancy.

Imaging findings in biopsy proven BAL are described in Table 2. The most notable finding is that the average SUV of lymph nodes with biopsy proven BAL was 7.9 \pm 2.2 . Despite the high FDG avidity, these lymph nodes were only mildly enlarged (short axis diameter of $1.2 \pm 0.3 \mathrm{~cm}$ ). Most patients $(95 \%)$ had multiple PET avid LNs $(3.8 \pm 1.3)$ and the vast majority of patients (90\%) had bilateral FDG avid lymph nodes.

In order to illustrate the clinical impact of non-invasive staging with PET or PET/CT, we compared radiologic stage with cytologic and histologic stage in the subset of patients with newly diagnosed NSCLC (Table 3). Six patients in our case series had a new diagnosis of NSCLC diagnosed by biopsy of a peripheral lung nodule. All patients received noninvasive staging with FDG PET/CT prior to invasive staging with EBUS-TBNA. For all 6 new diagnoses, radiologic staging suggested locally advanced non-surgical disease (Stage IIIB) based on increased uptake in contralateral mediastinal and/or hilar LNs (representative imaging in Figure 2). EBUS-TBNA identified BAL as an alternative explanation for the FDG avidity of all of these LNs (Table 3, Figure 1). In four of six patients, surgical resection of the primary lung cancer was performed 
Table 1 Patient demographics

\begin{tabular}{|c|c|}
\hline Demographics $(n=20)$ & Data \\
\hline Average age & $73 \pm 10$ \\
\hline \multicolumn{2}{|l|}{ Gender } \\
\hline Female & $6 / 20(30 \%)$ \\
\hline \multicolumn{2}{|l|}{ Country of Origin } \\
\hline Afghanistan & 1 \\
\hline China & 6 \\
\hline Korea & 1 \\
\hline Mexico & 3 \\
\hline Pakistan & 2 \\
\hline Russia & 1 \\
\hline Pakistan & 2 \\
\hline Columbia & 1 \\
\hline Iran & 1 \\
\hline Vietnam & 1 \\
\hline Peru & 1 \\
\hline USA & 2 \\
\hline Ever smoker & $50 \%$ \\
\hline Pack years & $32 \pm 19$ \\
\hline Known malignancy & $17 / 20$ \\
\hline
\end{tabular}

Table 2 Characteristics of lymph nodes

\begin{tabular}{lc}
\hline Imaging characteristics & Data \\
\hline Average maximum SUV of lymph node & $7.9 \pm 2.2$ \\
Average maximum short axis of lymph node $(\mathrm{cm})$ & $1.2 \pm 0.3$ \\
Average number of lymph nodes $>2$ SUV & $3.8 \pm 1.3$ \\
Bilateral PET Avid lymph nodes & $90 \%$ \\
\hline
\end{tabular}

SUV, standard uptake value.

with resection of lymph nodes. In all four cases surgical pathology confirmed the findings of EBUS TBNA cytology (Figure 3). As such, EBUS TBNA demonstrated a stage shift from a high clinical stage (based upon PET/CT) to a lower pathological stage (based on histology).

\section{Discussion}

In this case series we further characterize the demographic
Table 3 Radiological:Histological stage shift after EBUS-TBNA $(\mathrm{n}=6)$

\begin{tabular}{lcc}
\hline $\begin{array}{l}\text { Radiological } \\
\text { stage }(\mathrm{C})\end{array}$ & Histological stage (P) & LNs surgically resected \\
\hline IIIB & IA & Yes \\
IIIB & IIA & No \\
IIIB & IIB & No \\
IIIB & IA2 & Yes \\
IIIB & IA3 & Yes \\
IIIB & IIB & Yes \\
\hline
\end{tabular}

EBUS-TBNA, endobronchial ultrasound with transbronchial needle aspiration.

and radiographic features of BAL, an important cause of PET avidity in mediastinal and hilar lymph nodes. The striking finding in this series is the significantly elevated FDG uptake in lymph nodes with biopsy proven BAL. Our findings support the findings by several other groups who found the accumulation of anthracotic pigment within LNs can lead to highly FDG avid LNs on PET imaging. We observed a higher SUV max $(7.9 \pm 2.2)$ in our case series than in prior series. For example, Park et al. (8) described an average SUV max 5.8, Yilmaz et al. of 4.76 (13) and Korkmaz et al. (7) of 6.31. One notable feature is the range of values reported in these three series, from an SUV max of 1.2 to $31.9(7,8,13)$. Other demographic findings, including mean age, duration of tobacco exposure and male predominance in our study are similar to prior studies.

Multiple studies $(1,14-16)$ have sought to characterize the sensitivity and specificity of PET during the evaluation of mediastinal and hilar lymphadenopathy. Although no study has identified an SUV cut off that consistently discriminates a true positive (malignancy) from a false positive (nonmalignant disease), an SUV above 2.5 is generally thought to be highly suggestive of malignancy. In addition to SUV, the size of PET avid LNs is likely an important predictor of malignant or benign disease. In our series, the average size of anthracotic pigment laden LNs that were biopsied was $1.2 \pm 0.3 \mathrm{~cm}$. Toba et al. noted that PET avid LNs were more likely to represent a false positive if their mean short axis diameter was under $1 \mathrm{~cm}$ in size. Only 1/10 LN's were found to be false positive if measuring $>1 \mathrm{~cm}$ in size in their study. Most LN's representing false positive were found to have anthracosis or anthracosilicosis (17). This reported size is slightly smaller than in our series. It is unclear why this 

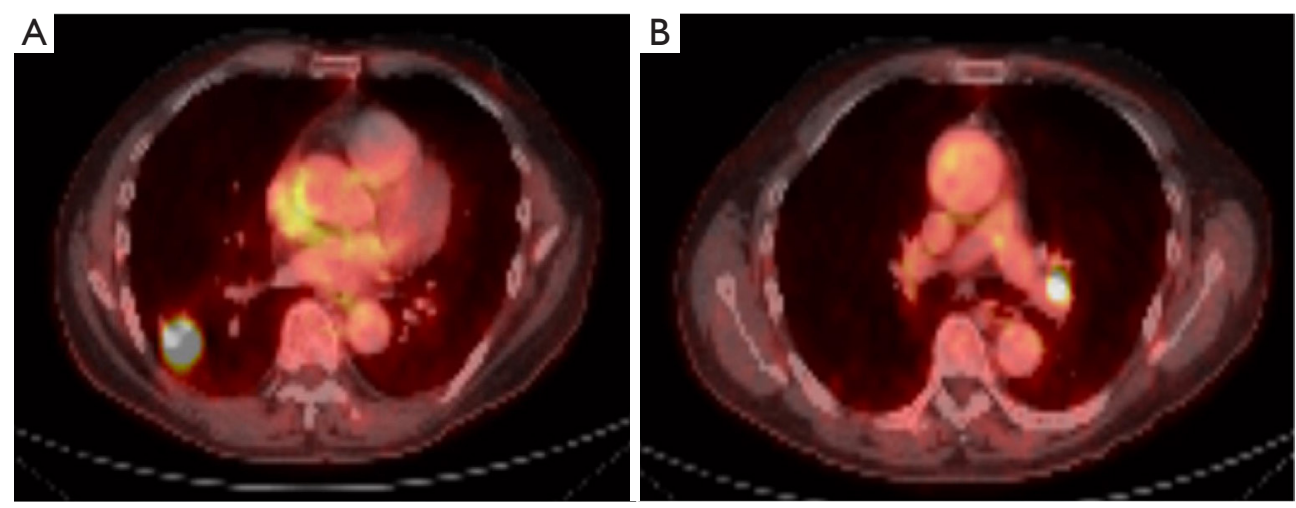

Figure 2 PET/CT demonstrating an FDG avid lung nodule with an FDG avid N3 lymph node. (A) FDG avid right lower lobe lung nodule. Pathology demonstrated NSCLC. (B) highly FDG avid N3 lymph node in the same patient. Pathology demonstrates benign anthracotic pigment.


Figure 3 Hematoxylin and eosin stained permanent sections of the level 6 lymph node biopsy showed an intact lymph node with abundant pigment filled macrophages in a fibrotic background. The nodal architecture is poorly preserved. There was no evidence of metastatic carcinoma or of lymphoma. (A) $10 \times$ magnification. (B) $40 \times$ magnification.

variability is noted, although measurement techniques and inherent differences in LN sizes at nodal stations may play a role.

In addition to $\mathrm{LN}$ size and SUV, the distribution of hilar and mediastinal LNs that are PET avid likely adds additional information regarding the likelihood of malignancy. Lymph node distribution is inconsistently identified in the published literature on BAL, and it is unclear if unilateral or bilateral LNs were PET avid in most of the published case series. Most series identify only LNs that were biopsied. In our study, LNs that were PET avid but were not biopsied (either due to LN position or to lack of clinical need) were also included in the description of bilateral $v s$. unilateral disease. There is some discrepancies in the reported literature regarding which LNs might become PET avid in the setting of BAL, but our data and others make clear that both hilar and mediastinal LNs may become PET avid in the setting of $\operatorname{BAL}(8,13,18)$. On average, we identified 3.8 PET avid LN's per patient with biopsy proven BAL, fitting with the hypothesis that inhaled particulate matter leads to a diffuse abnormality within the lung lymphatics. In addition, the distribution of PET avid LNs was bilateral in $90 \%$ of the cases in our case series. The absolute number and distribution of PET avid LNs was not well characterized in other case series $(7,8,13)$. In summary, key radiographic features that may differentiate lymph nodes with BAL from lymph nodes with metastatic disease include the smaller size and symmetric bilateral 
distribution of lymph nodes with BAL. This is in contrast to nodal metastases, which are more likely to be enlarged and asymmetric (Table 2).

Other case series that aim to describe imaging characteristics of BAL are from countries with high endemic biomass exposure, including Turkey and South Korea. Our series in notable for the international nature of the cohort and the first series from North America. Our institution in Northern California, cares for an international patient population, including many patients who have recently immigrated to the US. The striking demographic finding in this series is that $90 \%(18 / 20)$ of patients included in our series were born and raised outside of the United States. Countries of origin included Central and South America, Asia and the Middle East. One commonality between these countries is the more frequent use of biomass fuels. Biomass combustion, which includes combustion of wood, manure and other organic materials still serves as an important source for cooking and heating for over half of the world's population (19). We hypothesize that biomass exposure, which in some cases was supported by the patient's history, was the cause BAL observed in this case series.

Accurate staging dictates therapy for $\operatorname{NSCLC}(5,18,20)$. Six patients in this series had newly diagnosed NSCLC with PET avid contralateral mediastinal (N3) nodes. A representative PET/CT scan is shown in Figure 2. If the FDG uptake observed $(>2.5 \mathrm{SUV})$ in Figure $2 B$ were attributed to malignancy, based on $8^{\text {th }}$ edition TNM (11), these patients would be classified as stage IIIB, which would preclude a potentially curative surgical resection (Table 3). In contrast, invasive staging with EBUS showed that the PET avidity of these $\mathrm{N} 3$ nodes was due to BAL (Figure 1A,B). Four of these patients underwent surgical resection of the primary tumor as well as resection of the previously biopsied lymph nodes. All resected LNs showed an abundance of anthracotic pigment (Figure 3) without tumor, confirming the findings of the prior EBUS biopsy. Park et al. evaluated patients with potentially curative NSCLC with EBUSTBNA staging and evaluated the significant of anthracotic pigment within these specimens. The prevalence of malignant disease within these lymph nodes was $35.8 \%$. While $21.7 \%$ of lymph nodes in this study had anthracotic pigment, only 3 (4.9\%) lymph nodes had both anthracotic pigment and NSCLC present concomitantly (8). Park et al. describe a median SUV of anthracotic LNs of $4.4(1.7-11.6)$, and median LN size was $8.5(4.7-17.5) \mathrm{mm}$. These findings are similar to those demonstrated by our series.
Our study has several limitations. First, our referral base includes a large number of internationally born patients and our findings may not be generalizable to other populations. Additionally, only patients with both PET/ CT positive and anthracotic pigment on cytology were included in this study. It is possible that other patients with anthracotic pigment without positive PET scans exist. This represents a potential source of bias. In addition, case series do not give an estimate of prevalence, so it is unclear how common these radiographic findings may be. Finally, our retrospective case series is relatively small. The strengths of our study are the clear identification of a subset of patients at risk for radiographic overstaging, and the surgical confirmation of benign disease in four of our 6 cases of newly diagnosed NSCLC.

\section{Conclusions}

In this study, we describe the imaging characteristics of BAL, an important but potentially underrecognized cause of false-positive PET imaging in patients undergoing evaluation of malignancy. In our series, BAL was frequently bilateral, and led to multiple highly PET avid LNs without significant enlargement. Frequently, patients with biopsy proven BAL had a known exposure to inhaled biomass or urban airborne pollutants and the majority of them were from outside of North America. These results reinforce the current guideline recommendations for invasive mediastinal staging of PET avid LNs, especially if histologic sampling of a PET avid LN may provide an opportunity for a surgical cure of a newly diagnosed lung cancer.

\section{Acknowledgments}

Funding: This research was supported in part by the Nina Ireland Fund Grant.

\section{Footnote}

Reporting Checklist: The authors have completed the STROBE reporting checklist. Available at https://dx.doi. org/10.21037/jtd-21-142

Data Sharing Statement: Available at https://dx.doi. org/10.21037/jtd-21-142

Peer Review File: Available at https://dx.doi.org/10.21037/ jtd-21-142 
Conflicts of Interest: All authors have completed the ICMJE uniform disclosure form (available at https://dx.doi. org/10.21037/jtd-21-142). The authors have no conflicts of interest to declare.

Ethical Statement: The authors are accountable for all aspects of the work in ensuring that questions related to the accuracy or integrity of any part of the work are appropriately investigated and resolved. The study was conducted in accordance with the Declaration of Helsinki (as revised in 2013). The study was approved by institutional ethics committee of University of California San Francisco Office of Ethics and Compliance (IRB \#17-23634) and individual consent for this retrospective study was waived.

Open Access Statement: This is an Open Access article distributed in accordance with the Creative Commons Attribution-NonCommercial-NoDerivs 4.0 International License (CC BY-NC-ND 4.0), which permits the noncommercial replication and distribution of the article with the strict proviso that no changes or edits are made and the original work is properly cited (including links to both the formal publication through the relevant DOI and the license). See: https://creativecommons.org/licenses/by-nc-nd/4.0/.

\section{References}

1. Silvestri GA, Gonzalez AV, Jantz MA, et al. Methods for staging non-small cell lung cancer: Diagnosis and management of lung cancer, 3rd ed: American College of Chest Physicians evidence-based clinical practice guidelines. Chest 2013;143:e211S-50S.

2. Luketich JD, Schauer PR, Meltzer CC, et al. Role of positron emission tomography in staging esophageal cancer. Ann Thorac Surg 1997;64:765-9.

3. Escott EJ. Role of positron emission tomography/ computed tomography (PET/CT) in head and neck cancer. Radiol Clin North Am 2013;51:881-93.

4. Cheson BD, Fisher RI, Barrington SF, et al. Recommendations for initial evaluation, staging, and response assessment of Hodgkin and non-Hodgkin lymphoma: the Lugano classification. J Clin Oncol 2014;32:3059-68.

5. Shingyoji M, Nakajima T, Yoshino M, et al. Endobronchial ultrasonography for positron emission tomography and computed tomography-negative lymph node staging in non-small cell lung cancer. Ann Thorac Surg 2014;98:1762-7.
6. Mirsadraee M, Saffari A, Sarafraz Yazdi M, et al. Frequency of tuberculosis in anthracosis of the lung: a systematic review. Arch Iran Med 2013;16:661-4.

7. Korkmaz C, Demirbas S, Vatansev H, et al. The value of endobronchial ultrasound-guided transbronchial needle aspiration, 18-fluorodeoxyglucose positron emission tomography/computed tomography, and ultrasonography imaging techniques in the diagnosis of mediastinal and/ or hilar malignant, anthracotic, and other benign lymph nodes. Medicine (Baltimore) 2021;100:e24728.

8. Park YS, Lee J, Pang JC, et al. Clinical implication of microscopic anthracotic pigment in mediastinal staging of non-small cell lung cancer by endobronchial ultrasoundguided transbronchial needle aspiration. J Korean Med Sci 2013;28:550-4.

9. Mirsadraee M. Anthracosis of the lungs: etiology, clinical manifestations and diagnosis: a review. Tanaffos 2014;13:1-13.

10. El-Sherief AH, Lau CT, Wu CC, et al. International association for the study of lung cancer (IASLC) lymph node map: radiologic review with CT illustration. Radiographics 2014;34:1680-91.

11. Kandathil A, Kay FU, Butt YM, et al. Role of FDG PET/ CT in the Eighth Edition of TNM Staging of Non-Small Cell Lung Cancer. Radiographics 2018;38:2134-49.

12. Heinzerling AP, Guarnieri MJ, Mann JK, et al. Lung function in woodsmoke-exposed Guatemalan children following a chimney stove intervention. Thorax 2016;71:421-8.

13. Yilmaz Demirci N, Alici IO, Yilmaz A, et al. Risk factors and maximum standardized uptake values within lymph nodes of anthracosis diagnosed by endobronchial ultrasound-guided transbronchial needle aspiration. Turk J Med Sci 2015;45:984-90.

14. Toloza EM, Harpole L, McCrory DC, et al. Noninvasive staging of non-small cell lung cancer: a review of the current evidence. Chest 2003;123:137S-46S.

15. Shim SS, Lee KS, Kim BT, et al. Non-small cell lung cancer: prospective comparison of integrated FDG PET/ CT and CT alone for preoperative staging. Radiology 2005;236:1011-9.

16. Cerfolio RJ, Ojha B, Bryant AS, Raghuveer V, Mountz JM, Bartolucci AA. The accuracy of integrated PET-CT compared with dedicated PET alone for the staging of patients with nonsmall cell lung cancer. Ann Thorac Surg 2004;78:1017-23; discussion 1017-23.

17. Toba H, Kondo K, Otsuka H, et al. Diagnosis of the presence of lymph node metastasis and decision of 
operative indication using fluorodeoxyglucose-positron emission tomography and computed tomography in patients with primary lung cancer. J Med Invest 2010;57:305-13.

18. Rintoul RC, Tournoy KG, El Daly H, et al. EBUS-TBNA for the clarification of PET positive intra-thoracic lymph nodes-an international multi-centre experience. J Thorac Oncol 2009;4:44-8.

19. Bruce N, Perez-Padilla R, Albalak R, et al. Indoor air

Cite this article as: Ivanick NM, Shrestha P, Podolsky MJ, Walavalkar V, Lucas CH, Gesthalter YB, Seeley EJ. A retrospective observational study of benign anthracotic lymphadenitis and its association with PET avid lymph nodes in patients undergoing cancer evaluation. J Thorac Dis 2021;13(7):4228-4235. doi: 10.21037/jtd-21-142 pollution in developing countries: a major environmental and public health challenge. Bull World Health Organ 2000;78:1078-92.

20. Herth FJ, Eberhardt R, Krasnik M, et al. Endobronchial ultrasound-guided transbronchial needle aspiration of lymph nodes in the radiologically and positron emission tomography-normal mediastinum in patients with lung cancer. Chest 2008;133:887-91. 